\title{
Die quantitative Erfassung von Methan im Seewasser
}

\author{
Von Peter Bossard, Thomas Joller und Ernö Szabó \\ EAWAG, Dübendorf
}

Manuskript eingegangen am 25. April 1981

\begin{abstract}
The quantitative determination of methane in lake water

A preparation method for the quantitative analysis of methane in lake water samples has been developed. The method is based on the equilibrium reactions of gases in a closed two-phase (headspace-water) system, in which the headspace gas is analyzed gaschromatographically.

The method shows a standard deviation of $\pm 5 \%$, including sampling and sample preparation and can also be used for the quantitative determination of other dissolved gases.
\end{abstract}

\section{INHALTSVERZEICHNIS}

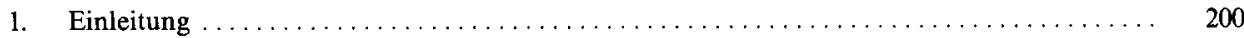

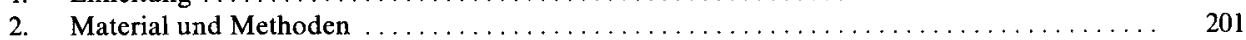

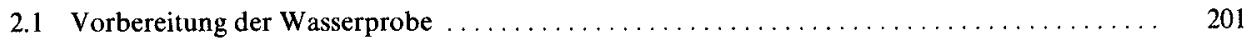

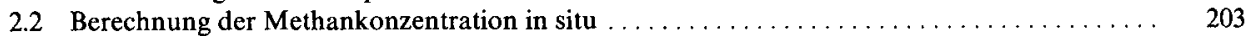

2.3 Erfassungsgrenzen für den quantitativen Nachweis von Methan $\ldots \ldots \ldots \ldots \ldots \ldots \ldots \ldots$

3. Reproduzierbarkeit der Methanmesswerte im Seewasser . . . . . . . . . . . . . . . . 206

4. Einfluss des Methankonzentrationsgradienten im See auf die Genauigkeit . . . . . . . . 207

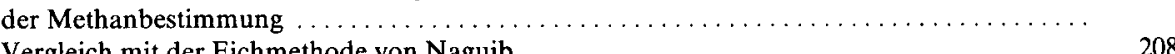

5. Vergleich mit der Eichmethode von Naguib . . . . . . . . . . . . . . . . . . . 208

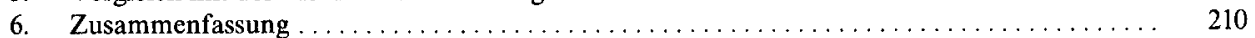

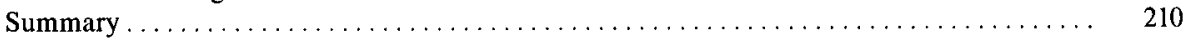

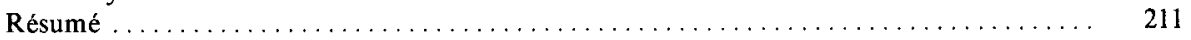

Literaturverzeichnis ....................................... 211

\section{Einleitung}

Die quantitative Erfassung von Methan im Seewasser ist vor allem in eutrophen Seen von Bedeutung. Im anaeroben Milieu ist Methan zwar ein biologisch inertes Gas. In der Grenzzone zwischen aeroben und anaeroben Wasserschichten jedoch kann die bakterielle Oxidation von akkumuliertem Methan eine bedeutende Sauerstoffsenke darstellen [3, 4]. Die Gaschromatographie ist ein zweckmässiges Instrument zur qualitativen und quantitativen Analyse von im Wasser gelöstem Methan. Da sich die direkte Injektion von Wasser in die Säule des Gaschromatographen (GC) in den meisten Fällen als ungeeignet erwies, haben verschiedene 
Autoren für limnologische Untersuchungen das sogenannte Strippingverfahren angewendet, bei welchem die im Wasser gelösten Gase mit einem Trägergas quantitativ vom Wasser getrennt werden. Die Gasbestimmung erfolgt sodann aus diesem abgetrennten Gasgemisch.

- Eine Wasserprobe wird in einem geschlossenen Behälter mit so viel Trägergas überschichtet, dass nach kräftigem Schütteln die im Wasser gelösten Gase quantitativ in die Gasphase übertreten [3, 5].

- Eine Wasserprobe wird direkt in den Trägergasstrom des GC vor der Trennsäule auf eine Glasfritte injiziert, wodurch das gelöste Gas ausgetrieben wird und direkt mit dem Trägergasstrom in die Säule gelangt [6].

Für beide Verfahren beträgt die zum quantitativen Ausstrippen ( $>97 \%$ ) benötigte Mindestmenge Trägergas das Volumen der Wasserprobe. Dabei gelangt das zu analysierende Methangas in einer relativ grossen Verdünnung in den GC. Die von Stainton [5] und Rudd [3] beschriebene Methode bedingt einen mehrmaligen Transfer der Wasser- bzw. Gasprobe in verschiedene Gefässe, was sich auf die Genauigkeit der quantitativen Bestimmung nachteilig auswirken muss. Die von Swinnerton [6] entwickelte Methode begünstigt eine Verschleppung der zu analysierenden Gase während des Trennvorganges in der Säule, wodurch die Messgenauigkeit ebenfalls beeinträchtigt wird.

In der vorliegenden Arbeit wurde zur routinemässigen Bestimmung kleinster Methankonzentrationen im Wasser auf eine Strippingmethode verzichtet. Die im folgenden vorgestellte Methode basiert auf dem Lösungsgleichgewicht von Methan zwischen Wasser und Kopfgas in einem geschlossenen Behälter wie die von Naguib [2] entwickelte Bestimmungsmethode. Im Gegensatz zur Naguibschen Methode wird hier jedoch die Berechnung von Gleichgewichtskonzentrationen nicht mit einer eleganten Eichmethode umgangen, um zur Bestimmung der In-situ-Gaskonzentration zu gelangen. Die hier vorgestellte Methode liefert somit die theoretischen Grundlagen zur Naguibschen Methode.

\section{Material und Methoden}

\subsection{Vorbereitung der Wasserprobe}

Die Wasserproben aus einer Van-Dorn-Schöpfflasche wurden auf dem See in 300-ml-Infusionsflaschen eingefüllt. Zu diesem Zweck wurde der Flasche ein «Kragen» aufgesteckt (siehe Abb.1). Die Flasche wurde mit einem Schlauch blasenfrei gefüllt, indem das mehrfache Volumen der Glasflasche mit Probenwasser ausgespült und die Flaschen anschliessend mit einem Schraubverschluss mit Gummiseptum verschlossen wurden. Der aufgesteckte Kragen setzte dabei die Flaschenöffnung unter Wasser und erleichterte das blasenfreie Verschliessen unter Luftabschluss. Vor dem Verschliessen wurde durch das überstehende Wasser $1 \mathrm{ml}$ konz. NaOH zur Fixierung der Probe zugegeben, um die bakterielle Methanoxidation in der Flasche zu verhindern. Die so fixierten Wasserproben konnten mehr als eine Woche aufbewahrt werden, ohne dass sich ihre Methankonzentration veränderte. 


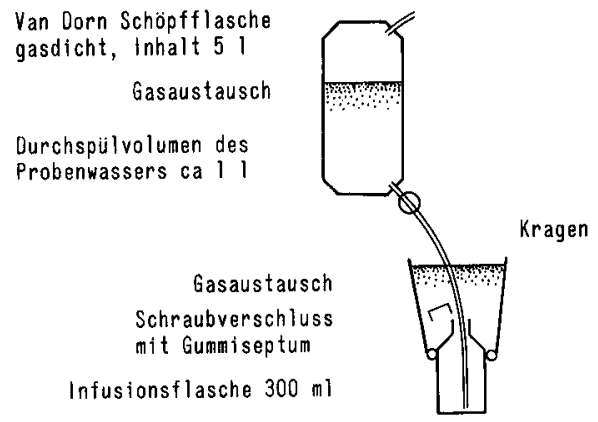

Abb. 1. Probenahme für Methananalysen.

Figure 1. Sampling for the quantitative determination of dissolved methane in lake water.

$1=$ Van Dorn sampling bottle (volume 51 ), gastight. $2=$ Volume of the flushed sampled water: ca. 11 .

$3=$ Collar placed on an infusion bottle facilitating bubble-free closing of the bottle (volume $300 \mathrm{ml}$ ).

Die blasenfrei abgefüllte Wasserprobe wurde im Labor auf Raumtemperatur aufgewärmt, gewogen und sodann mit $30 \mathrm{ml}$ Trägergas (Helium, Stickstoff oder Luft) überschichtet, indem bei der Injektion des Trägergases $30 \mathrm{ml}$ Wasser durch eine offene Kanüle aus der Flasche verdrängt wurden (Abb.2).

$\mathrm{Zu}$ diesem Zweck wurde die Flasche waagrecht gelegt, die mit Trägergas gefüllte Spritze durch das Gummiseptum gestossen und ein leichter Überdruck in der Flasche erzeugt, bis sich das Septum leicht wölbte. In diesem Moment wurde eine zweite Kanüle in das Septum gestossen, welche bei der Gasinjektion das Entweichen des Wassers erlaubte. Dabei war darauf zu achten, dass keine Gasblasen mit dem austretenden Wasser entwichen. Nach erfolgtem Druckausgleich (Innendruck = Aussendruck) wurde auch die offene Kanüle entfernt und die Probe während 20 Minuten geschüttelt. Dadurch stellte sich ein Lösungsgleichgewicht ein zwischen dem im Wasser gelösten und dem im Kopfgas vorhandenen Methan. Zur Bestimmung des Kopfgas- bzw. Wasservolumens wurde die Infusionsflasche ein zweites Mal gewogen.

Bei bekannter Wassertemperatur, bekanntem Barometerdruck, Gas- und Wasservolumen kann durch gaschromatographische Bestimmung des Partialdruckes von

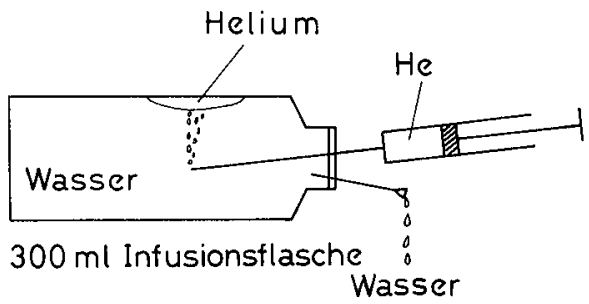

Abb. 2. Vorbereitung der Wasserprobe für Methananalyse.

Figure 2. Preparation of the water sample for methane headspace analysis.

$1=$ Carrier gas (Helium or air) is injected under pressure into the infusion bottle. $2=\mathrm{A}$ syringe needle allows the escape of the displaced water. 
Methan im Kopfgas die ursprüngliche Methankonzentration in der Wasserprobe berechnet werden.

Zur Methananalyse diente ein Gaschromatograph der Carlo Erba, Modell Fractovap 2101, mit Flammenionisationsdetektor (Porapak-Q-Säule, 100-120 mesh von $2 \mathrm{~m}$ Länge). Aus dem Kopfgas der Wasserprobe wurde mit einer Spritze 0,4 ml Gas entnommen. Davon wurde $0,25 \mathrm{ml}$ in den Gaschromatographen injiziert. Um den bei mehreren Gasentnahmen entstehenden leichten Unterdruck im Kopfgas der Flasche zu kompensieren, wurde eine Spritze mit verschliessbarem Ventil verwendet. Der leichte Unterdruck störte das Lösungsgleichgewicht in der Flasche nicht, solange diese nicht geschüttelt wurde, wie wiederholte Messungen aus der gleichen Flasche zeigten (Streuung $< \pm 2 \%$ ).

Aus der gemessenen Peakhöhe des Methans wurde durch Vergleich mit der Peakhöhe von kalibriertem Eichmethan der Partialdruck des Methans im Kopfgas der Wasserprobe berechnet. Wie entsprechende Vergleichsmessungen zeigten, verhalten sich die Höhe und die darunterliegende Fläche (Integrationswert) eines Peaks bei $\mathrm{CH}_{4}$-Partialdrücken $<2 \%$ absolut linear. Bei höheren Partialdrücken ist die Linearität nicht mehr gewährleistet, da die Peakhöhen mit zunehmendem $\mathrm{CH}_{4}$-Partialdruck zu kleine Werte anzeigen. So zeigt reines Methan 10-15\% kleinere Peakhöhen an als die korrespondierenden Peakflächen, welche sich über alle Konzentrationsbereiche linear zur entsprechenden Konzentration verhalten.

Nach erfolgter Analyse wurde die Infusionsflasche geöffnet und die Wassertemperatur mit einem Quecksilberthermometer gemessen.

\subsection{Berechnung der Methankonzentration in situ}

Die nachfolgende Berechnung kann durch Modifikation der gasspezifischen Konstanten für jedes Gas $\mathrm{i}$ angewendet werden. In einer geschlossenen Flasche, welche aus den zwei Phasen «Kopfgas» und «Wasser» besteht (Abb. 3 b), befinden sich sämtliche Gase in einem Lösungsgleichgewicht, welches für ein Gas i durch das Gesetz von Henry $\mathrm{C}_{\mathrm{is}}=\mathrm{K}_{\mathrm{Hi}} \cdot \mathrm{p}_{\mathrm{i}}$ definiert ist.

$\mathrm{C}_{\text {is }}[\mathrm{Mol} / \mathrm{l}] \quad$ Gleichgewichtskonzentration des Gases i im Wasser

$\mathrm{K}_{\mathrm{Hi}}\left[\frac{\mathrm{Mol}}{1 \cdot \mathrm{atm}}\right] \begin{aligned} & \begin{array}{l}\text { Henry-Konstante des Gases } \mathrm{i} \text { (abhängig von der Temperatur } \\ \text { und dem Gas } \mathrm{i} \text { ) }\end{array} \\ & \text { and }\end{aligned}$ $\mathrm{p}_{\mathrm{i}}$ [atm] Partialdruck des Gases i im Kopfgas

Wird in einer blasenfrei abgefüllten Flasche (Abb.3a) ein Teil des Wassers durch ein Trägergas (Kopfgas) ersetzt (Abb.3b), so ist zur Zeit $t_{0} p_{i}=0$ und $C_{i s}=C_{i}$. Durch Schütteln wird $C_{i s}<C_{i}$, und $p_{i}$ nimmt im Laufe der Zeit zu, gemäss der Reaktionsgleichung erster Ordnung.

$$
\frac{d p_{i}}{d t}=\lambda\left(p_{i s}-p_{i}\right), \quad \text { bis } \quad p_{i}=p_{i s} \quad \text { wird, }
$$

für:

$\mathrm{p}_{\text {is }}$ [atm] Partialdruck des Gases $\mathrm{i}$ im Kopfgas, welche der Gleichgewichtskonzentration des Gases i im Wasser entspricht

$\lambda[1 / \mathrm{t}] \quad$ Reaktionskonstante 


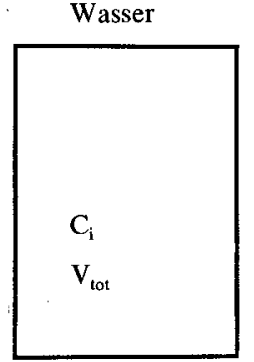

a) Vor,
Wasser + Gas

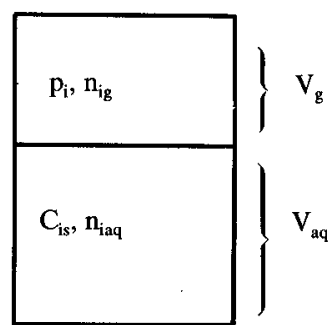

b) nach Zugabe des Kopfgases.

$$
\mathrm{V}_{\mathrm{tot}}=\mathrm{V}_{\mathrm{g}}+\mathrm{V}_{\mathrm{aq}}
$$$$
\mathrm{C}_{\mathrm{i}} \cdot \mathrm{V}_{\mathrm{aq}}=\mathrm{n}_{\mathrm{ig}}+\mathrm{n}_{\mathrm{iqq}}
$$

\section{$\mathrm{C}_{\mathrm{i}}[\mathrm{Mol} / \mathrm{l}, \mathrm{mg} / \mathrm{l}]=$ In situ-Konzentration des Gases i im Wasser.}

$\mathrm{V}_{\mathrm{g}}, \mathrm{V}_{\mathrm{aq}}, \mathrm{V}_{\text {tot }}[\mathrm{l}]=$ Volumen des Kopfgases $\left(\mathrm{V}_{\mathrm{g}}\right)$, des Wassers $\left(\mathrm{V}_{\mathrm{aq}}\right)$, Volumen der blasenfrei abgefüllten Flasche $\left(\mathrm{V}_{\text {to }}\right)$.

$$
\mathrm{n}_{\mathrm{ig}}, \mathrm{n}_{\mathrm{iaq}}[\mathrm{Mol}]=\text { Anzah1 Mol des Gases i im Kopfgas }\left(\mathrm{n}_{\mathrm{ig}}\right) \text {, im Wasser }\left(\mathrm{n}_{\mathrm{iqq}}\right) \text {. }
$$

a) Before, $b$ ) after addition of headspace carrier gas. Parameters: $\mathrm{C}_{\mathrm{i}}=$ in situ concentration of gas $\mathrm{i}$ in lake water. $C_{\text {is }}=$ equilibrium concentration of gas $i$ in water in a two-phase system (water headspace). $p_{i}=$ partial pressure of gas $i$ in headspace. $n=$ number of moles of gas $i$ in gas phase $\left(n_{i g}\right)$ and in water phase $\left(n_{\text {iaq }}\right) . V=$ volume. $p=$ atmospheric pressure. $p_{i s}=$ partial pressure of gas $i$ in headspace corresponding to equilibrium concentration $\mathrm{C}_{\text {is }}$ in the water phase.

Abb. 3. Schematische Darstellung der physikalischen Parameter in der Wasserprobe.

Figure 3. Schematic description of physical parameter in the water sample.

Für die Berechnung der In-situ-Konzentration des Gases i gilt:

$$
\mathrm{C}_{\mathrm{i}}\left[\frac{\mathrm{Mol}}{\mathrm{l}}\right]=\frac{\mathrm{n}_{\mathrm{ig}}+\mathrm{n}_{\mathrm{iaq}}}{\mathrm{V}_{\mathrm{aq}}} \text {. }
$$

Daraus lässt sich unter Berücksichtigung des Gesetzes für ideale Gase $(p \cdot V=n \cdot R \cdot T)$ und des Gesetzes von Henry folgende Gleichung für $C_{i}$ ableiten:

$$
\mathrm{C}_{\mathrm{i}}\left[\frac{\mathrm{Mol}}{1}\right]=\left(\frac{\mathrm{p}_{\mathrm{i}} \cdot \mathrm{V}_{\mathrm{g}}}{\mathrm{R} \cdot \mathrm{T} \cdot \mathrm{V}_{\mathrm{aq}}}+\mathrm{K}_{\mathrm{Hi}} \cdot \mathrm{p}_{\mathrm{i}}^{\prime}\right) \cdot \mathrm{P} .
$$

Für $\mathrm{C}_{\mathrm{i}}$ in [mg/l] und für $a_{\mathrm{i}}=\mathrm{K}_{\mathrm{Hi}} \cdot \mathrm{f}$ gilt:

$$
\mathrm{C}_{\mathrm{i}}\left[\frac{\mathrm{mg}}{\mathrm{l}}\right]=\mathbf{P} \cdot \mathrm{p}_{\mathrm{i}}^{\prime} \cdot \mathrm{k} \cdot \mathrm{MG}\left(\frac{\mathrm{V}_{\mathrm{g}}}{\mathbf{R} \cdot \mathrm{T} \cdot \mathrm{V}_{\mathrm{aq}}}+\frac{a_{\mathrm{i}}}{\mathrm{f}}\right),
$$

für:

$\mathrm{P} \quad[\mathrm{atm}] \quad$ Barometerdruck

$\mathrm{p}_{\mathrm{i}}^{\prime} \quad[\mathrm{atm} / \mathrm{atm}] \quad$ Partialdruck des Gases i im Kopfgas

$\mathrm{R} \quad\left[\frac{1 \cdot \mathrm{atm}}{\mathrm{Mol} \text { Kelvin }}\right]$ 0,08205 (universelle Gaskonstante)

$\mathrm{T}$ [Kelvin] Absolute Temperatur 


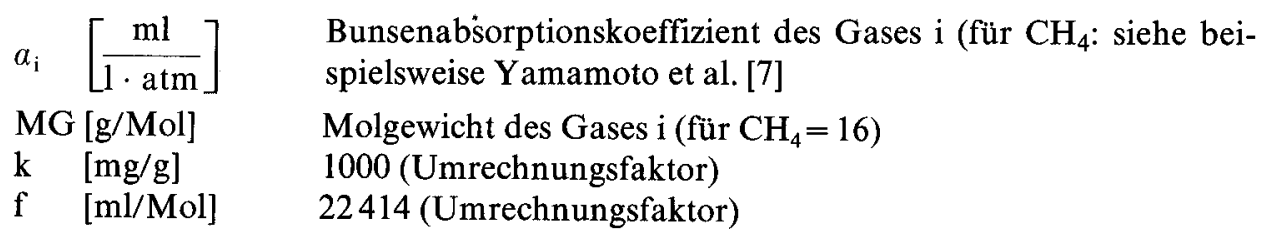

Zur Berechnung der In-situ-Konzentration eines Gases i wird Gleichung (3) verwendet.

\subsection{Erfassungsgrenze für den quantitativen Nachweis von Methan}

Bei der maximal möglichen Geräteempfindlichkeit des GC wurden für die berechneten In-situ-Methankonzentrationen von 0,3 bis $1,0 \mu \mathrm{g} / 1$ Peakhöhen von 4,5 bis $14 \mathrm{~mm}$ notiert. Die Erfassungsgrenze für quantitativ auswertbare Methankonzentrationen mit einer Streuung von $\pm 5 \%$ dürfte ungefähr bei ca. $1 \mu \mathrm{g} \mathrm{CH}_{4} / 1$ in situ liegen. Eine Senkung der Erfassungsgrenze könnte durch eine Verkleinerung des Kopfgas-Wasser-Verhältnisses $\left(\mathrm{V}_{\mathrm{g}} / \mathrm{V}_{\mathrm{aq}}\right.$ viel kleiner als 0,1$)$ in der Flasche erreicht werden. Wie Tabelle 1 zeigt, kann diese jedoch höchstens noch um das Vierfache gesteigert werden. Aus der Tabelle ist ebenfalls ersichtlich, dass bei einem $V_{g} / V_{a q}=1$, wie es beim Strippingverfahren üblich ist, der Partialdruck von Methan im Kopfgas $7,5 \mathrm{mal}$ kleiner ist als beim hier verwendeten Verhältnis von 0,1 .

Bei einer Verkleinerung von $\mathrm{V}_{\mathrm{g}} / \mathrm{V}_{\mathrm{aq}}$ muss jedoch beachtet werden, dass die Genauigkeit der Methanbestimmung auch von der Genauigkeit der Bestimmung von $\mathrm{V}_{\mathrm{g}}$ abhängt $\left(\mathrm{V}_{\mathrm{g}} / \mathrm{V}_{\mathrm{g}}=\mathrm{a}\right.$ beträgt bei der gravimetrischen Bestimmung der Volumina $\mathrm{V}_{\text {tot }}-\mathrm{V}_{\mathrm{aq}}=\mathrm{V}_{\mathrm{g}}$ in der Infusionsflasche ca. $1 \mathrm{ml}$ ).

Im weiteren sollte $\mathrm{V}_{\mathrm{g}}$ viel grösser sein als die für die Injektion in den Gaschromatographen benötigte Totalmenge $\left(V_{I}\right)$, damit sich das Lösungsgleichgewicht von Methan zwischen Gas- und Wasserphase während der wiederholten Gasentnahmen nicht in gravierender Weise verschiebt. $\left(V_{I}=1,5 \mathrm{ml}\right.$ reicht für 4-5 Injektionen in den GC aus und bewirkt bei einem $\mathrm{V}_{\mathrm{g}}$ von $30 \mathrm{ml}$ eine Erniedrigung des Kopfgasdruckes von $5 \%$. Wenn die Infusionsflasche zwischen den einzelnen Gasinjektionen nicht geschüttelt wird und wenn eine Injektionsspritze mit verschliessbarem Ventil verwendet wird, wirkt sich die Erniedrigung des Kopfgasdruckes nicht unmittelbar auf die Höhe des Methanpeaks im GC aus, wie entsprechende Untersuchungen zeigten, da anscheinend bei der molekularen Diffusion des gelösten Methans in die Gasphase die Reaktionskonstante $\lambda$ ziemlich klein sein muss.)

Tabelle 1. Einfluss von $\mathrm{V}_{\mathrm{g}} / \mathrm{V}_{\text {aq }}$ auf den Gleichgewichtspartialdruck von Methan $\left(\mathrm{p}_{\mathrm{CH}_{4}}\right)$ im Kopfgas bei $20^{\circ} \mathrm{C}$ und Atmosphärendruck, ausgehend von einer In-situ-Konzentration $\left(\mathrm{C}_{\mathrm{i}}\right)$ von $1 \mathrm{mg} \mathrm{CH}_{4} / 1$.

Table 1. Infiuence of $\mathrm{V}_{\mathrm{g}} / \mathrm{V}_{\mathrm{aq}}$ on the equilibrium partial pressure of methane $\left(\mathrm{p}_{\mathrm{CH}_{4}}\right)$ in headspace at $20^{\circ} \mathrm{C}$ and $1.0 \mathrm{~atm}$ atmospheric pressure. Initial in situ concentration (before headspace addition) $\mathrm{C}_{\mathrm{i}}=1 \mathrm{mg}$ $\mathrm{CH}_{4} / 1$.

Comment: The table shows that $\mathrm{p}_{\mathrm{CH}_{4}}$ at $\mathrm{V}_{\mathrm{g}} / \mathrm{V}_{\mathrm{aq}}=1$ is 7.5 times smaller than $\mathrm{p}_{\mathrm{CH}_{4}}$ at $\mathrm{V}_{\mathrm{g}} / \mathrm{V}_{\mathrm{aq}}=0.1$.

\begin{tabular}{lllr}
\hline $\mathrm{V}_{\mathrm{g}} / \mathrm{V}_{\mathrm{aq}}$ & $\mathrm{p}_{\mathrm{CH}_{4}}$ in atm & $\mathrm{V}_{\mathrm{g}} / \mathrm{V}_{\mathrm{aq}}$ & $\mathrm{p}_{\mathrm{CH}_{4}}$ in atm \\
\hline $10^{-6}$ & $42,3 \times 10^{-3}$ & 0,1 & $11,3 \times 10^{-3}$ \\
$1 \times 10^{-3}$ & $41,2 \times 10^{-3}$ & 1 & $1,5 \times 10^{-3}$ \\
$1 \times 10^{-2}$ & $33,2 \times 10^{-3}$ & & \\
\hline
\end{tabular}




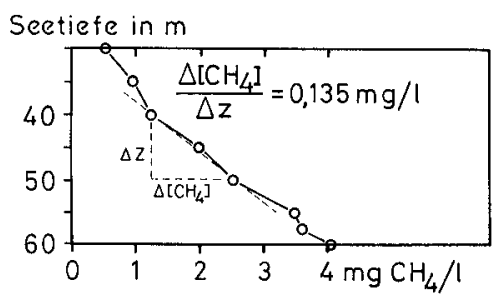

Abb.4. Methankonzentrationen im vertikalen Seeprofil des Baldeggersees: Berechnung des

Methankonzentrationsgradienten in $45 \mathrm{~m}$ Tiefe.

Figure 4. Methane concentrations in a vertical profile of Lake Baldegg: Estimation of the methane concentration gradient in $45 \mathrm{~m}$ below water surface. $\mathrm{z}=$ depth in meters.

Trotzdem muss damit gerechnet werden, dass für $\mathrm{V}_{\mathrm{g}}<30 \mathrm{ml}$ demnach mit grösseren Streuungen bei der Methanbestimmung gerechnet werden muss, als im nachfolgenden Kapitel 3 angegeben wird.

\section{Reproduzierbarkeit der Methanmesswerte im Seewasser}

Um die Streuung der gemessenen Methankonzentrationen in einer bestimmten Seetiefe zu ermitteln, wurden vier Schöpfflaschenproben aus $45 \mathrm{~m}$ Tiefe des Baldeg-

Tabelle 2. Reproduzierbarkeit bei der Bestimmung von Methankonzentrationen im Seewasser.

\begin{tabular}{lllll}
\hline $\begin{array}{l}\text { Schöpf- } \\
\text { flasche }\end{array}$ & $\begin{array}{l}\text { Infusions- } \\
\text { flasche }\end{array}$ & $\mathrm{mg} \mathrm{CH}_{4} / 1$ & $\mathrm{mg} \mathrm{CH} / 1$ & $\begin{array}{r}\mathrm{mg} \mathrm{CH}_{4} / 1 \\
\text { Durchschnitt } \\
\text { pro Seetiefe }\end{array}$ \\
$\mathrm{S}$ & $\mathrm{I}$ & $\overline{\mathrm{I}}$ & $\overline{\mathrm{S}}$ & \\
\hline 1 & 11 & $1,98 \pm 1,1 \%$ & $1,98 \pm 0,64 \%$ & \\
2 & 12 & $1,97 \pm 1,0 \%$ & & \\
& 13 & $2,05 \pm 0,5 \%$ & $2,01 \pm 2,4 \%$ & \\
3 & 14 & $1,98 \pm 1,4 \%$ & & \\
4 & 15 & $2,06 \pm 0,6 \%$ & $2,05 \pm 0,76 \%$ & \\
& 16 & $2,04 \pm 1,4 \%$ & & \\
\hline
\end{tabular}

$\overline{\mathrm{I}}=$ Durchschnittswert von 3 bis 4 Injektionen pro Infusionsflasche. Der prozentuale Fehler ist auf die Streuung bei der Bestimmung der einzelnen Peakhöhen zurückzuführen.

$\mathrm{S}=$ Durchschnittswert von 2 Infusionsflaschen pro Schöpfflasche. Der prozentuale Fehler beinhaltet unter anderem auch die Streuung bei der Bestimmung der Volumina $V_{g}$ und $V_{a q}$.

Aufgrund der Fehlerfortpflanzung ist jede Messung pro Infusionsflasche mit einem Fehler von $\pm 5 \%$ behaftet.

Table 2

Mean deviations in methane determinations:

$\mathrm{S}=$ Of repeated sampling with Van Dorn bottle at the same lake depth.

$\mathbf{I}=$ Of several infusion bottles filled from the same Van Dorn bottle.

$\overline{\mathbf{I}}=$ Mean value of 3 to 4 injections per infusion bottle into the GC.

$\underline{\bar{S}}=$ Mean value of 2 infusion bottles per sampling bottle (Van Dorn bottle).

$\overline{\mathrm{T}}=$ Mean value per lake depth. 
gersees in je zwei Infusionsflaschen abgefüllt. Ihre Methankonzentration wurde nach der oben beschriebenen Methode bestimmt. Dazu wurden aus jeder Infusionsflasche 3-4 Injektionen à $0,25 \mathrm{ml}$ Kopfgas zur gaschromatographischen Analyse durchgeführt. Tabelle 2 zeigt die Mittelwerte der bestimmten Methankonzentrationen

- pro Infusionsflasche $(\overline{\mathrm{I}})$,

- pro Schöpfflasche $(\overline{\mathrm{S}})$,

- pro Seetiefe $(\overline{\mathrm{F}})$ inkl. Streuung (s) in \%.

Es fällt dabei auf, dass die $\mathrm{CH}_{4}$-Konzentration der zweiten Infusionsflasche aus der gleichen Schöpfflasche durchwegs tiefer ist als diejenige der ersten. Diese systematische Abweichung ist nur teilweise mit dem im See vorhandenen vertikalen Methankonzentrationsgradienten erklärbar (siehe Abb.4), und dies auch nur unter der Voraussetzung, dass sich der Inhalt in der Schöpfflasche nicht durchmischt. Pro Abfüllmenge von etwa einem Liter pro Infusionsflasche ergibt dies bei einem Innendurchmesser der Schöpfflasche von $12 \mathrm{~cm}$ eine vertikale Wassersäule von $9 \mathrm{~cm}$. Die gemessenen Methankonzentrationen in den zwei Infusionsflaschen stellen somit die Mittelwerte zweier übereinanderliegender Wasserpakete dar. Der durch lineare Interpolation ermittelte $\mathrm{CH}_{4}$-Gradient beträgt $0,135 \mathrm{mg} / 1 \cdot \mathrm{m}$. Die zweite Infusionsflasche sollte somit $12 \mu \mathrm{g} / 1$ weniger Methan aufweisen als die erste der gleichen Schöpfflasche. Der restliche Unterschied in der Methankonzentration muss auf Verluste durch Manipulation zurückgeführt werden.

\section{Einfluss des Methankonzentrationsgradienten im See auf die Genauigkeit der Methanbestimmung}

In Abb. 5 werden einige typische vertikale Methankonzentrationsprofile aus dem Lungernsee, dem Greifensee und dem Baldeggersee gezeigt, wie sie sich gegen Ende der Stagnationsperiode präsentieren.

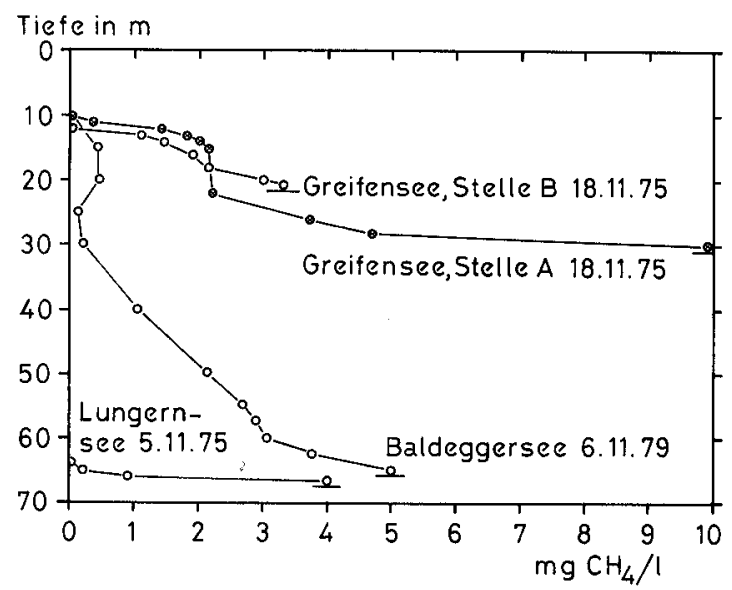

Abb. 5. Methankonzentrationen im Tiefenprofil dreier Schweizer Seen, Ende Stagnation.

Figure 5. Methane concentrations in vertical profiles of three Swiss lakes at the end of summer stagnation (Greifensee: two sample sites, $A$ and B). $z=$ depth in meters. 
Die beschriebene Methode zur quantitativen Bestimmung von Methan im Seewasser weist im Falle des präsentierten Beispiels eine maximale Streuung von $\pm 5 \%$ auf. Daraus ist abzuleiten, dass beim berechneten Methankonzentrationsgradienten von $0,135 \mathrm{mg} / \mathrm{l} \cdot \mathrm{m}$ im Baldeggersee (siehe Abb.4) in $45 \mathrm{~m}$ Tiefe die Schöpfflasche bei wiederholter Probenahme um weniger als $\pm 75 \mathrm{~cm}$ geschwankt haben darf. In Sedimentnähe sind die Methankonzentrationsgradienten der in Abb. 4 dargestellten Profile wesentlich höher (Greifensee in $30 \mathrm{~m}$ Tiefe: $2,6 \mathrm{mg} / 1 \cdot \mathrm{m}$; Lungernsee in $66,5 \mathrm{~m}$ Tiefe: $3,1 \mathrm{mg} / 1 \cdot \mathrm{m}$; Baldeggersee in $63 \mathrm{~m}$ Tiefe: $0,5 \mathrm{mg} / 1 \cdot \mathrm{m}$ ) als im vorgenannten Beispiel. Wenn bei solchen Gradienten durch die Probenahme allein keine grössere Streuung als $\pm 5 \%$ verursacht werden soll, muss beispielsweise im Greifensee die Schöpfflasche in $30 \mathrm{~m}$ Tiefe auf $\pm 17 \mathrm{~cm}$ genau eingestellt werden können. Dies ist jedoch ohne Hilfsmittel kaum möglich.

Diese Beispiele zeigen, dass die Genauigkeit der Methanbestimmung in erster Linie von der Probenahmetechnik und erst in zweiter Linie von der Aufbereitung und Analyse der Probe abhängt.

Rudd [4] installierte für seine Untersuchungen in einem $10 \mathrm{~m}$ tiefen See ein vertikales steifes Rohr, welches am Seegrund verankert wurde. Daran befestigt, führten mehrere Plastikschläuche in die verschiedenen Tiefen. Mit dieser permanenten Einrichtung wurden die Wasserproben an die Oberfläche gepumpt. Eine solche Einrichtung ist jedoch für tiefere Seen aus technischen Gründen kaum möglich. Von den Autoren wurde eine nicht ganz so genaue, aber wesentlich einfachere Probenahmetechnik angewendet. An der Schöpfflasche wurde mit einer Leine ein Lot so befestigt, dass es genau $50 \mathrm{~cm}$ unter der Schöpfflasche plaziert war. Bei der Probenahme wurde das Lot, welches etwa das Gewicht der leeren Schöpfflasche hatte, vorsichtig auf das Sediment aufgesetzt. Mit Hilfe des Zählwerkes an der Winde konnte nun die Schöpfflasche mit ziemlich grosser Genauigkeit in die gewünschte Seetiefe (in Sedimentnähe) gehoben werden.

\section{Vergleich mit der Eichmethode von Naguib}

Die von Naguib [2] angewandte Methode zur Methanbestimmung beruht ebenfalls auf einem Gleichgewichtsverhältnis zwischen Wasser- und Gasphase. Naguib verzichtet jedoch auf eine Berechnung von Gleichgewichtskonzentrationen, indem er eine Eichmethode anwendet, welche ihm den direkten Vergleich der im Gaschromatographen gemessenen Peakflächen der Eichproben mit den Seewasserproben ermöglicht. Zu diesem Zweck stellt er eine mit Methan gesättigte Wasserlösung her, die er für eine Verdünnungsreihe verwendet. Aus den gemessenen Peakflächen der Methanproben der Verdünnungsreihe erstellt er eine Eichkurve.

Diese Bestimmungsmethode setzt eine absolut gleiche Behandlung aller Wasserproben voraus. Druck, Temperatur, Wasservolumen und Kopfgasvolumen müssen in allen Probeflaschen genau gleich gross ein. Eventuelle Abweichungen können ohne Berechnung der Gleichgewichtskonzentrationen nicht erfasst werden.

Im folgenden wurden solche Verdünnungsreihen aus methangesättigten Wasserlösungen hergestellt, und deren Methankonzentration wurde in Prozenten der Methansättigung nach Bossard-Joller-Szabó bestimmt (Abb.6). Innerhalb einer Streuung von $\mathrm{s}=8 \%$ verläuft die Beziehung linear. Dabei ist zu bemerken, dass das Herstellen 
Anteil methan- $\%$ gesättigtes Wasser in der Wasserprobe (Eichprobe)

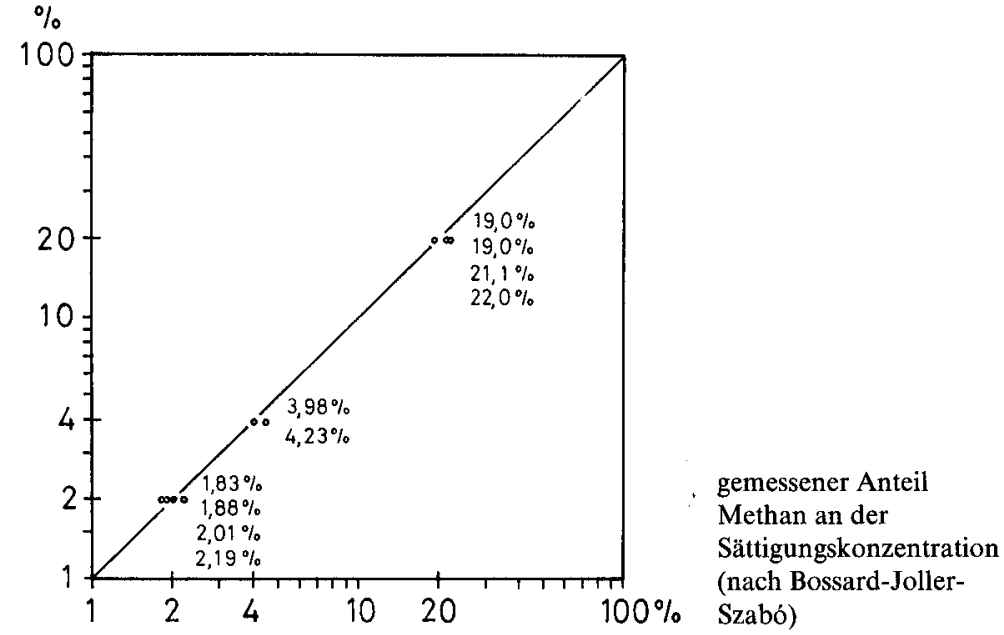

Abb.6. Direkter Vergleich zwischen der Methode nach Naguib [2] und der Methode nach BossardJoller-Szabó.

Figure 6. Direct comparison between the Naguib [2] method and the method after Bossard-Joller-Szabó.

einer Verdünnungsreihe mit methangesättigtem Wasser wegen der leichten Flüchtigkeit des Methangases (Blasenbildungen) nicht ganz problemlos ist und dass insbesondere bei Verdünnungsfaktoren $>50$ die Streuungen ziemlich gross sein können.

Die Sättigungskonzentration berechnet Naguib aus physikalischen Tabellen (z. B. d'Ans-Lax [1]). Zur Berechnung benötigt er die Temperatur der Eichlösung und den Druck des überstehenden Kopfgases (bei Innendruck = Aussendruck entspricht dies dem Barometerdruck). Ein Vergleich zwischen tabellarisch berechneten Sättigungskonzentrationen (a, Tab.3, berechnet nach Yamamoto et al. [7]) und den nach Bossard-Joller-Szabó gemessenen und berechneten Methankonzentrationen der gesättigten Lösungen (b, Tab.3) lässt ersehen, dass bei der Herstellung einer methangesättigten Lösung und bei deren Vorbereitung zur gaschromatographischen Bestimmung mit einer gewissen Streuung zu rechnen ist.

Aus dem direkten Vergleich der beiden Methoden kann geschlossen werden, dass

Tabelle 3. Methansättigungskonzentrationen in Wasser: a) tabellarisch ermittelt nach Yamamoto et al. [7], b) gemessen und berechnet nach Bossard-Joller-Szabó.

Table 3. Methane saturation concentrations in water: a) computed from tables after Yamamoto et al. [7], b) measured and determined after Bossard-Joller-Szabó.

\begin{tabular}{lllr}
\hline $\begin{array}{l}\text { Druck } \\
\text { in Torr }\end{array}$ & $\begin{array}{l}\text { Temperatur } \\
\text { in }{ }^{\circ} \mathrm{C}\end{array}$ & $\begin{array}{l}\text { Methankonzentration in } \mathrm{mg} \mathrm{CH}_{4} / 1 \\
\text { a) tabellarisch } \\
\text { ermittelt }\end{array}$ & $\begin{array}{r}\text { b) gemessen und } \\
\text { berechnet nach } \\
\text { Bossard-Joller-Szabó }\end{array}$ \\
\hline 770 & 25,5 & 22,6 & 22,7 \\
770 & 18,4 & 26,0 & 27,0 \\
\hline
\end{tabular}


die theoretischen Grundlagen der hier präsentierten Berechnungsmethode korrekt sind.

Die Methode nach Naguib hat den Vorteil, dass zur Ermittlung von Methankonzentrationen keine komplexen Formeln benötigt werden. Sie bedingt jedoch einen zusätzlichen analytischen Aufwand, da bei jeder Messreihe eine Eichkurve bestimmt werden muss, welche äusserste Sorgfalt voraussetzt.

Bei der Methode nach Bossard-Joller-Szabó erfolgt die Eichung mit handelsüblichem kalibriertem Methangas, was keinen analytischen Mehraufwand verursacht. Im weiteren kann auf eine Normierung der Volumenverhältnisse zwischen Wasservolumen und Kopfgasvolumen verzichtet werden, was die Probenahme etwas vereinfacht. Der dadurch bedingte grössere Berechnungsaufwand fällt bei einer computerisierten Auswertung nicht ins Gewicht.

\section{Zusammenfassung}

Es wurde eine Aufbereitungsmethode entwickelt, welche die quantitative gaschromatographische Bestimmung von Methan nach dem Prinzip der Gleichgewichtsreaktionen von Gasen in einem geschlossenen Zweiphasensystem (Wasser-Kopfgas) erlaubt. Die einfache Vorbereitung der Proben minimiert manipulationsbedingte Fehlerquellen. Diese Methode weist inklusive Probenahme eine Streuung von ca. $\pm 5 \%$ auf. Die Erfassungsgrenze für eine quantitative Auswertung liegt bei ca. $1 \mu \mathrm{g}$ $\mathrm{CH}_{4} / \mathrm{l}$.

Ein direkter Vergleich dieser Methode mit einer von Naguib [2] entwickelten Eichmethode bestätigt die Richtigkeit der theoretischen Berechnungsgrundlagen und deren Eignung für den praktischen Gebrauch.

Der Einfluss des vertikalen Methankonzentrationsgradienten im See auf die Genauigkeit der Methanbestimmung wird diskutiert: Bei Gradienten von über $0,5 \mathrm{mg}$ $\mathrm{CH}_{4} / 1 \cdot \mathrm{m}$ ist in erster Linie die Genauigkeit der Probenahme verantwortlich. Methankonzentrationsprofile aus drei voralpinen Seen zeigen, dass der Gradient der Methankonzentrationen im Bereich der untersten Meter über dem Seesediment auf $2-3 \mathrm{mg} \mathrm{CH} / 4 \cdot \mathrm{m}$ ansteigen kann.

Die Methode eignet sich auch zur quantitativen Erfassung anderer im Wasser gelöster Gase.

\section{RÉSUMÉ}

La détermination quantitative du méthane dans l'eau des lacs

Une méthode de préparation, pour les échantillons d'eau du lac, a été développée, laquelle permet l'analyse quantitative du méthane par la chromatographie en phase gazeuse, selon les règles des réactions d'équilibre des gaz, dans un système fermé à deux phases (eau-gaz). La simplicité de la préparation des échantillons minimise les erreurs de manipulation. La méthode montre une déviation standard de $\pm 5 \%$, y compris la prise d'échantillons. La concentration minimale qui peut être analysée quantitativement est de environ $1 \mu \mathrm{g} \mathrm{CH}_{4} / 1$.

Une comparaison directe de cette méthode avec la méthode de calibration développée par Naguib [2] ne montre pas de différences significatives et confirme la valeur de son application pratique.

L'influence du gradient de concentration du méthane dans les lacs sur l'exactitude de la détermination du méthane est discutée. Avec des gradients de plus de $0,5 \mu \mathrm{g} \mathrm{CH}_{4} / 1 \cdot \mathrm{m}$, l'exactitude de la prise d'échantillon 
est le facteur déterminant. Le profile de concentration du méthane dans trois lacs des préalpes montre que le gradient vertical de concentration peut monter de $2-3 \mu \mathrm{g} \mathrm{CH} / 1 \cdot \mathrm{m}$ dans les premiers mètres audessus du sédiment.

La méthode peut aussi être utilisée pour la détermination quantitative d'autres gaz dissous dans l'eau.

\section{SUMMARY}

The quantitative determination of methane in lake water

A preparation method for lake water samples has been developed, which allows the quantitative gaschromatographic analysis of methane according to the rules of equilibrium reactions of gases in a closed two-phase (headspace-water) system.

A simple sample preparation minimizes manipulation artifacts. The method shows a standard deviation of $\pm 5 \%$, including sampling and'sample preparation. The minimal in situ concentration enabling a quantitative analysis is $1 \mu \mathrm{g} \mathrm{CH} / 4$.

A direct comparison of this method with a calibration method developed by Naguib [2] shows no significant differences and supports the validity of the applied formulas for practical use. The influence of the vertical in situ methane concentration gradient in lakes on the accuracy of the methane determination is discussed: At gradients of more than $0.5 \mathrm{mg} \mathrm{CH}_{4} / 1 \cdot \mathrm{m}$ the accuracy of the sampling technique is the most critical factor. Concentration profiles of three prealpine lakes show that vertical concentration gradients in the lower hypolimnion may rise to $2-3 \mathrm{mg} \mathrm{CH}_{4} / \mathrm{l} \cdot \mathrm{m}$.

This method can also be used for the quantitative determination of other dissolved gases.

\section{LITERATURVERZEICHNIS}

1 d'Ans-Lax: Taschenbuch für Chemiker und Physiker, Bd. 1. Berlin, Heidelberg 1967.

2 Naguib, M.: A rapid method for quantitative estimation of dissolved methan and its application in ecological research. Arch. Hydrobiol. 82/1+4, 60-73 (1978).

3 Rudd, J.W., Hamilton, R.D., und Campbell, N. E.: Measurement of microbial oxidation of methane in lake water. Limnol. Oceanogr. 19/3, 519-524 (1974).

4 Rudd, J.W., und Hamilton, R.D.: Two samplers monitoring dissolved gases in lake water and sediments (Note). Limnol. Oceanogr. 20/5, 902-906 (1975).

5 Stainton, M.P.: A syringe gas-stripping procedure for gaschromatographic determination of dissolved inorganic and organic carbon in fresh water and carbonates in sediments. J. Fish. Res. Bd Can. 30, 1441-1445 (1973).

6 Swinnerton, J.W., Linnenbom, V.J. und Cheek, C. H.: Determination of dissolved gases in aqueous solutions by gas chromatography. Analyt. Chem. 34/4, 483-485 (1962).

7 Yamamoto, S., Alcauskas, J.B., und Crozier, T.E.: Solubility of methane in distilled water and seawater. J. Chemical Engng Data 21/1, 78 80 (1976).

Adressen der Autoren: Dr. P. Bossard, dipl. sc.nat. Th. Joller, Seenforschungslaboratorium der EAWAG/ETH, CH-6047 Kastanienbaum, Schweiz - E. Szabó, dipl. Chem., EAWAG, CH-8600 Dübendorf, Schweiz. 